\title{
miR-30a suppresses lung cancer progression by targeting SIRT1
}

\author{
Yaowu Guan ${ }^{1}$, Zhongming Rao ${ }^{1}$ and Cheng Chen ${ }^{2}$ \\ ${ }^{1}$ Department of Thoracic Surgery, Zhumadian Central Hospital, Zhumadian, Henan 463000, China \\ ${ }^{2}$ Department of Radiotherapy, Nanjing Medical University Affiliated Cancer Hospital, Cancer Institute of Jiangsu Province, \\ Nanjing, Jiangsu 210009, China \\ Correspondence to: Yaowu Guan, email: gyw0396@sina.cn \\ Cheng Chen, email: njmudoctor@163.com \\ Keywords: miR-30a; SIRT1; proliferation; apoptosis; invasion \\ Abbreviations: SIRT1: silent information regulator 1; miRNA: microRNA \\ Received: September 15, $2017 \quad$ Accepted: December 04, $2017 \quad$ Published: December 21, 2017 \\ Copyright: Guan et al. This is an open-access article distributed under the terms of the Creative Commons Attribution License 3.0 \\ (CC BY 3.0), which permits unrestricted use, distribution, and reproduction in any medium, provided the original author and source \\ are credited.
}

\section{ABSTRACT}

The class III histone deacetylase silent information regulator 1 (SIRT1) is frequently overexpressed in a variety of tumors, including lung cancer; however, its regulatory mechanisms are largely unknown. In this study, we found that an inconsistent trend between SIRT1 protein and mRNA levels in human lung cancer tissues, suggesting that a post-transcriptional mechanism may involved in SIRT1 regulation. Because microRNAs are important post-transcriptional regulators of gene expression, candidate miRNAs that could potentially bind SIRT1 were gained through bioinformatics analyses. We further experimentally validated SIRT1 as the direct target of miR-30a by evaluating SIRT1 expression in lung cancer cells after the overexpression or knockdown of $\mathrm{miR}-30 \mathrm{a}$ and by luciferase assay. Moreover, we showed that miR-30a inhibited proliferation, invasion and promoted apoptosis of lung cancer cells by inhibiting SIRT1 in vitro and in vivo. Taken together, this study identified a new regulatory axis in which miR-30a and SIRT1 regulate the proliferation, invasion and apoptosis of lung cancer cells and lung tumorigenesis.

\section{INTRODUCTION}

Lung cancer is the most frequently diagnosed and leading cause of death cancer worldwide [1], and nonsmall-cell lung cancer (NSCLC) accounts for $75-80 \%$ of all cases [2]. Most lung cancer patients are diagnosed with metastatic and advanced disease, and only a small proportion is eligible for surgical resection and radical treatment [3]. Thus, new treatment strategies are needed, and further understanding the molecular mechanisms underlying lung carcinogenesis is of major significance and might provide novel strategies for lung cancer treatment.

Sirtuins are belong to the class III histone deacetylase (HDAC) family and have been linked to longevity in lower organisms and to mammalian metabolism [4, 5]. Sirtuin 1 (SIRT1) functions by deacetylating histone (e.g. H4-Lys16 and H3-Lys9) and non-histone proteins(e.g. Ku70 and p300) in an NAD ${ }^{+}$ dependent manner, thus modifying gene expression and modulating protein activity $[6,7]$. SIRT1 plays important roles in numerous processes, including cell cycle, metabolism, DNA repair, aging and cell survival under stress conditions $[4,5]$.

Importantly, dysregulation of SIRT1 has been demonstrated in various cancers including prostate, breast, ovarian and lung cancers, implicating a pathogenetic role for SIRT1 in malignancies [8-11]. SIRT1 can function as an oncogene by regulating the acetylation of some tumor suppressors, including p53 [12] and FoxOs [13]. However, other studies also suggest that SIRT1 may also have tumor-suppressive function in some mouse model $[14,15]$. The precise role of SIRT1 in cancer may depend on the specific cell or tumor type. Though up-regulation of SIRT1 has been observed in lung cancer [11], the roles of SIRT1 in the initiation and progression of lung cancer remain poorly understood.

SIRT1 expression can be regulated at the transcriptional level. For example, tumor suppressors p53 and HIC1 (hypermethylated in cancer 1) can suppress 
SIRT1 transcription through binding to SIRT1 promoter region $[16,17]$. However, this is not the unique mechanism for dysregulation of SIRT1 in tumors. For example, HuR, a RNA binding protein, stabilizes SIRT1 mRNA through 3'-untranslated region (3'-UTR) interactions leading to increased SIRT1 levels (19). This suggests that the posttranscriptional regulation of SIRT1 may also be significant in governing SIRT1 expression in lung cancer.

MiRNAs are small non-coding RNAs of 20 22 nucleotides which can lead to mRNA degradation and/or translational repression repress gene expression through interactions with the 3 '-untranslated regions (3'-UTRs) of target gene transcripts [18-20]. Through this mechanism, miRNAs can regulate a great variety of biological processes, including cell proliferation, differentiation, migration, apoptosis, development and metabolism [21-23]. MiR-30a has been reported significantly downregulated in lung cancer [24], indicating that miR-30a may play an important role in tumorigenesis and development of lung cancer. However, the function of miR-30a, especially in lung cancer, remains unclear.

In this study, we showed that SIRT1 as a direct target gene of miR-30a, resulting in the downregulation of SIRT1 protein expression. The potential role of miR$30 \mathrm{a}$ as an anti-oncogene of lung cancer through SITR1 targeting in proliferation, invasion and apoptosis had been experimentally validated in vitro and in vivo.

\section{RESULTS}

\section{The upregulation of SIRT1 protein but not mRNA in lung cancer tissues}

We first determined the SIRT1 protein levels in 6 pairs of clinical lung cancer tissues. We found that SIRT1 protein levels were consistently upregulated in lung cancer tissues (Figure 1A and 1B). Subsequently, we performed quantitative RT-PCR to check the levels of SIRT1 mRNA in the same 6 pairs of cancerous and noncancerous tissues. However, SIRT1 mRNA levels did not significantly differ between cancerous and noncancerous tissues (Figure 1C). The disparity between SIRT1 protein and mRNA levels in lung cancer tissues suggested a post-transcriptional mechanism involved in the regulation of SIRT1.

\section{Prediction of SIRT1 as a target of miR-30a}

One common post-transcriptional regulation is the suppression of mRNA transcripts by miRNAs. To identify which miRNAs can potentially target SIRT1 in lung cancer cells, a list of predicted miRNAs which may target SIRT1 was compiled using three computational algorithms, TargetScan [25], miRanda [26] and PicTar [27]. Among the candidates, miR-30a is a tumor suppressor that is frequently downregulated in lung cancer [28, 29]. The predicted interactions between miR-30a and the targeting sites within the 3'-UTR of SIRT1 are illustrated in Figure 2A.

\section{Detection of an inverse correlation between miR- $30 a$ and SIRT1 levels in lung cancer tissues}

Based on the opposite expression patterns between miRNAs and their target genes, we next investigated whether miR-30a was inversely correlated with SIRT1 in clinical patient tissues. After measuring the miR-30a levels in 6 pairs of lung cancer tissues and noncancerous tissues, we observed that miR-30a was indeed downregulated in lung cancer tissues (Figure 2D). We suggested that SIRT1 was a target of miR-30a, based on both computational predictions and the inverse correlation between miR-30a and SIRT1 levels in human lung cancer tissues.

\section{Validation of SIRT1 as a direct target of miR-30a}

Two human lung cancer cell lines (A549 and H1975) was used to further confirm the direct correlation between miR-30a and SIRT1 after overexpression or knockdown of miR-30a. As expected, the cellular levels of miR-30a were significantly increased in A549 and H1975 cells transfected with miR-30a mimics and decreased dramatically when cells were transfected with miR-30a inhibitor (Figure 3A). Consequently, the expression of SIRT1 protein was significantly inhibited by the overexpression of miR-30a in A549 and H1975 cells, while the miR-30a inhibitor significantly increased the SIRT1 protein levels in lung cancer cells (Figure 3B and $3 \mathrm{C}$ ). To determine at what level miR-30a influenced SIRT1 expression, we repeated the above experiments and examined the expression of SIRT1 mRNA after transfection. Overexpression or knockdown of miR-30a did not affect SIRT1 mRNA levels (Figure 3D).

A luciferase reporter assay was performed to confirm whether the negative regulatory effects of miR$30 \mathrm{a}$ on SIRT1 expression were mediated by the binding of miR-30a to the predicted sites in the 3'-UTR of SIRT1 mRNA and inhibit SIRT1 expression. The full-length 3'UTR of SIRT1, containing the presumed miR-30a binding sites, was placed downstream of the firefly luciferase gene in a reporter plasmid. Then the recombination plasmid was transfected into $293 \mathrm{~T}$ and A549 cells along with pre-miR30a. As expected, the luciferase activity was dramatically reduced in cells co-transfected with luciferase reporter plasmid and miR-30a mimics (Figure 3E and 3F). Then we introduced point mutations into the binding site of the SIRT1 3'-UTR to eliminate the predicted miR-30a binding site. This mutated luciferase reporter was unaffected through either the overexpression or knockdown of miR30a (Figure 3E and 3F). This finding suggested that the binding sites strongly contribute to the interaction between miR-30a and SIRT1 mRNA. In conclusion, our results demonstrate that miR-30a directly recognizes and binds to the 3 '-UTR of the SIRT1 transcript and inhibits SIRT1 translation. 


\section{The role of miR-30a in regulating SIRT1 in lung cancer cells}

We next analyzed the biological function of miR-30a-driven repression of SIRT1 expression in lung cancer cells. Because SIRT1 is known to induce cell proliferation [30] and invasion [31] and suppress apoptosis [32], miR-30a may suppress SIRT1 expression to affect cell proliferation, invasion and apoptosis. As expected, overexpression of miR-30a in A549 cells showed decreased cell viability and invasion and increase cell apoptosis; in contrast, inhibiting miR-30a had an opposite effect on lung cell proliferation, invasion and apoptosis (Figure 4A, 4B, 4D, 4F, 4G and 4H). Efficient overexpression of SIRT1 in A549 cells was achieved (Supplementary Figure 1A-1F). As expected, overexpression of SIRT1 in A549 cells indeed caused increased cell proliferation, invasion and decreased cell apoptosis (Supplementary Figure 2A-2E). More importantly, proliferation, invasion and apoptosis assays revealed that ectopic expression of miR-30a-resistant SIRT1 dramatically attenuated the inhibitory effect of the miR-30a on cell proliferation, invasion and apoptosis (Figure 4C, 4E, 4F, 4G and 4H). Taken together, our results suggest that SIRT1 is crucial to the proliferation, invasion and apoptosis of lung cancer cells and that miR$30 \mathrm{a}$ might suppress proliferation and invasion and promote apoptosis in lung cancer cells by silencing SIRT1.

\section{The function of miR-30a-SIRT1 axis on lung cancer growth in vivo}

We next evaluated the effects of miR-30a and SIRT1 on the growth of lung cancer xenograft in mice. A549 cells were infected with the lentiviral plasmid to express miR-30a. Efficient overexpression of miR-30a and inhibition of SIRT1 was shown in Supplementary Figure 3A-3B. The A549 cells $\left(1 \times 10^{6}\right.$ cells per $\left.0.1 \mathrm{~mL}\right)$ were

A

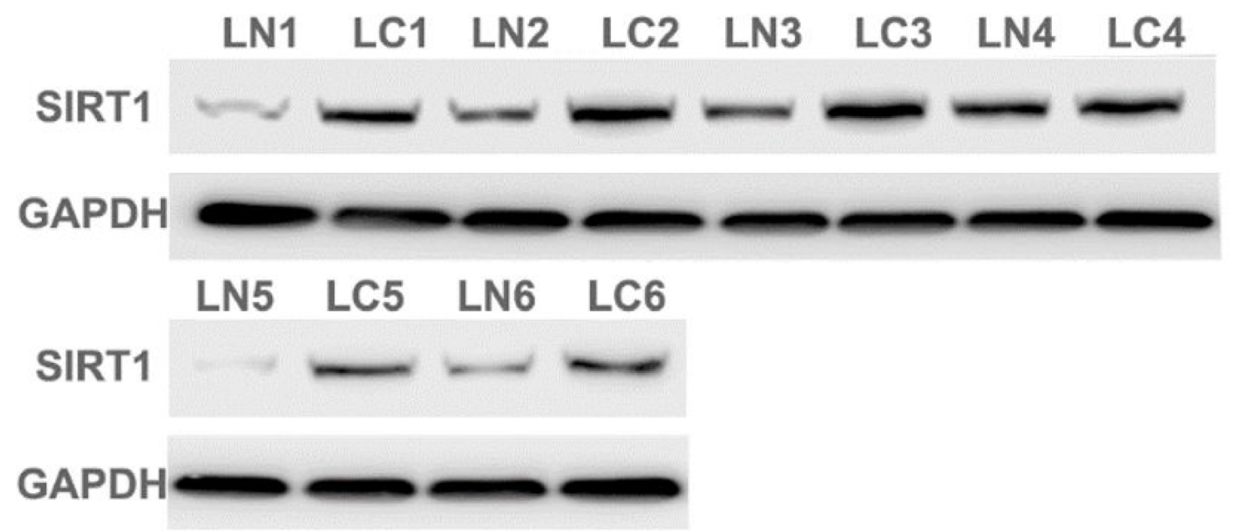

B
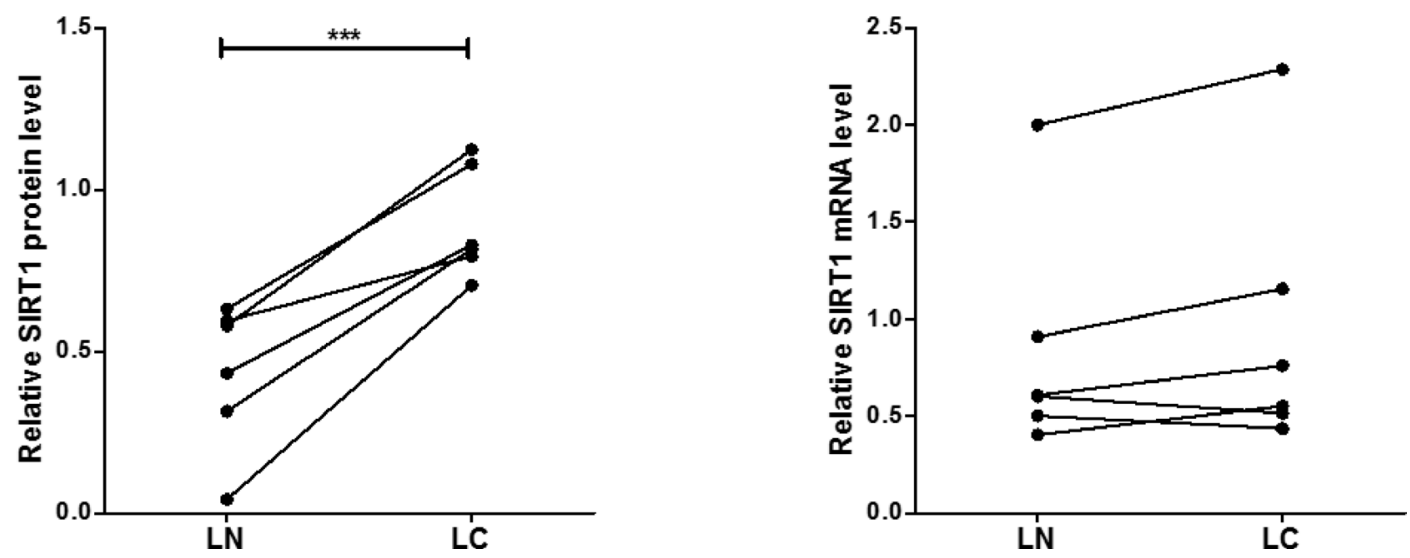

Figure 1: SIRT1 protein and mRNA expression levels in lung cancer tissues. (A and B) Western blot analysis of the expression of the SIRT1 protein in 6 pairs of lung cancer (LC) and normal adjacent (LN) tissue samples. A: representative image; B: quantitative analysis. (C) Quantitative RT-PCR analysis of the relative expression levels of SIRT1 mRNA in 6 pairs of LC and LN samples. ${ }^{* * *} P<0.001$. 

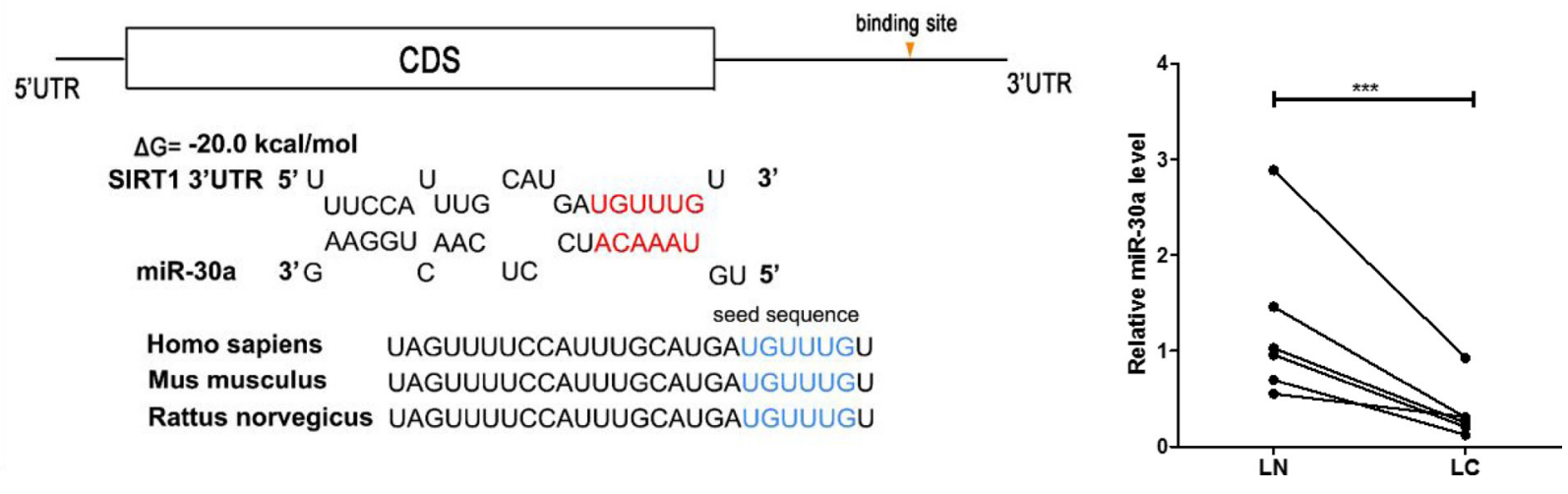

Figure 2: Prediction of the miR-30a binding site within the SIRT1 3'-UTR. (A) Schematic depicting the hypothetical duplexes formed through interactions between the binding sites in the SIRT1 3'-UTR (top) and miR-30a (bottom). The predicted free energy of each hybrid is indicated. The seed recognition sites are denoted, and all nucleotides in these regions are highly conserved across species. (B) Quantitative RT-PCR analysis of the miR-30a expression levels in six pairs of LC and LN samples. ${ }^{* * *} P<0.001$.

A

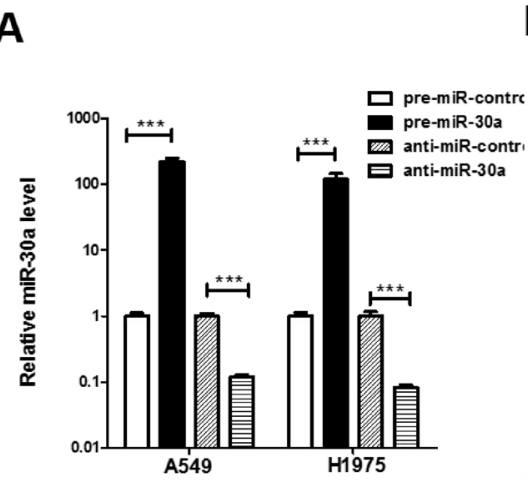

D

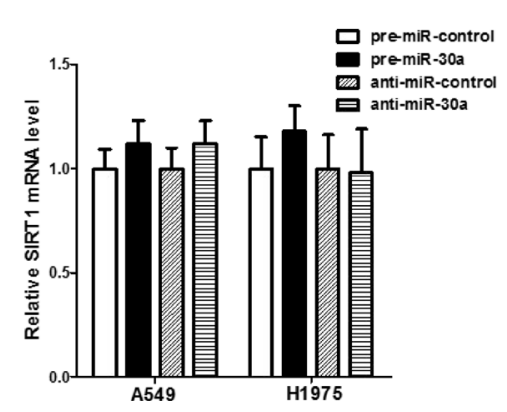

B

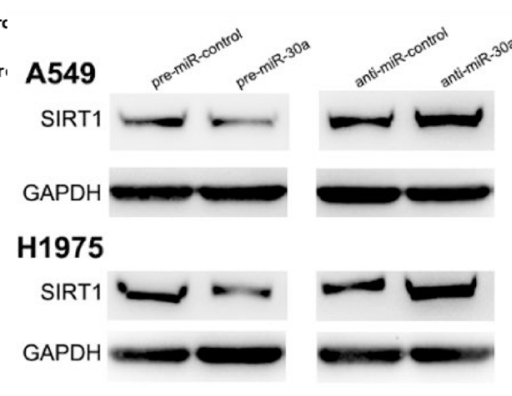

E

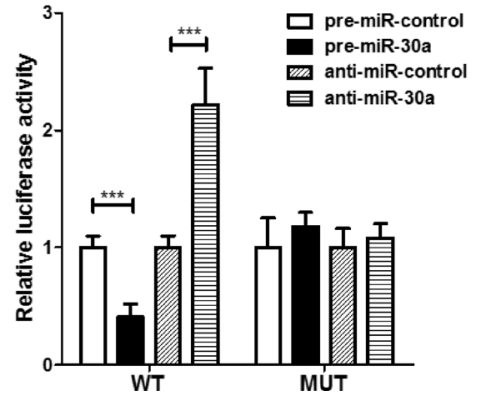

C

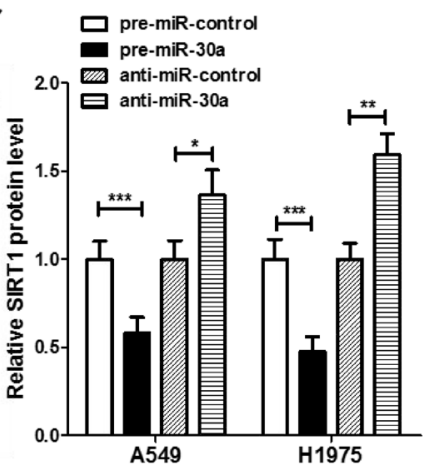

$\mathbf{F}$

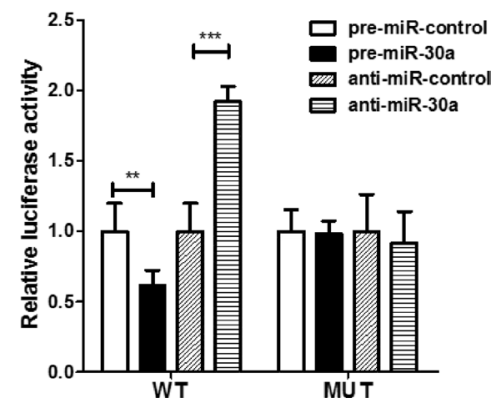

Figure 3: Direct post-transcriptional regulation of SIRT1 expression through miR-30a. (A) Quantitative RT-PCR analysis of the expression levels of miR-30a in A549 and H1975 cells transfected with equal doses of the miR-30a mimic (pre-miR-30a), miR-30a inhibitor (anti-miR-30a) or scrambled negative control RNA (pre-miR-control or anti-miR-control). (B and C) Western blotting analysis to detect SIRT1 protein levels in A549, and H1975 cells transfected with equal doses of the miR-30a mimic, miR-30a inhibitor or scrambled negative control RNA. (B) representative image; (C) quantitative analysis. (D) Quantitative RT-PCR analysis of SIRT1 mRNA levels in A549 and H1975 cells transfected with equal doses of the miR-30a mimic, miR-30a inhibitor or scrambled negative control RNA. (E and F) Direct recognition of the SIRT1 3'-UTR by miR-30a. Firefly luciferase reporters containing either wild-type (WT) or mutant (MUT) miR-30a binding sites in the SIRT1 3'-UTR were co-transfected into 293T (E) and A549 (F) cells with equal doses of the miR-30a mimic, miR-30a inhibitor or scrambled negative control RNA. After twenty-four hours post-transfection, the cells were assayed using a luciferase assay kit. Firefly luciferase values were normalized to $\beta$-galactosidase activity, and the results were calculated as the ratio of firefly luciferase activity in the miR-30a-transfected cells normalized to the negative control RNA-transfected cells. The results are presented as the mean \pm S.E. of three independent experiments. $\left({ }^{*} p<0.05 ;{ }^{* *} p<0.01 ;{ }^{* * *} p<0.005\right)$. 
infected with/without the miR-30a lentiviral expression plasmid (miR-30a), SIRT1 plasmid(SIRT1), or miR-30a lentiviral expression plasmid plus SIRT1 overexpression plasmid(miR-30a+SIRT1); then, the cells were implanted subcutaneously into 6-week-old nude mice and tumor growth was measured after 25 days. A significant decrease in the sizes and growth of tumors was observed in the miR-30a-overexpressing group compared with the control group, whereas the sizes and weights in the tumors from the group implanted with the SIRT1-overexpression plasmid were dramatically increased (Figure 5A-5C). Additionally, SIRT1 overexpression attenuated the suppressive effect of miR-30a on tumor growth (Figure 5A-5C), suggesting that miR-30a might inhibit tumor growth by silencing SIRT1. After 25 days, the miR-30aoverexpression group show a significant increase in the expression of miR-30a compared with tumors from control group (Figure 5D). Tumors from miR-30a-overexpressing group displayed reduced SIRT1 protein levels compared with tumors from control group, whereas the tumors of SIRT1-overexpressing group showed elevated SIRT1 protein levels (Figure 5E). Tumors with both miR30a plus SIRT1 overexpression exhibited significantly higher levels of SIRT1 compared to tumors with miR-

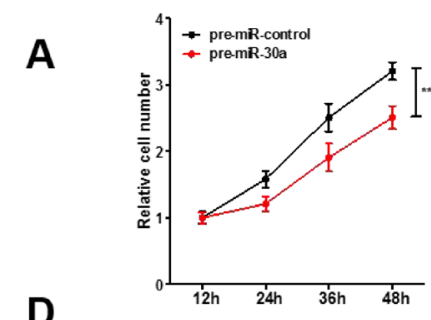

D

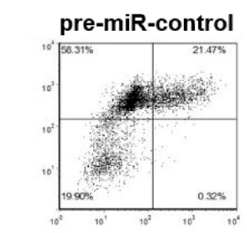

E pre-miR-control
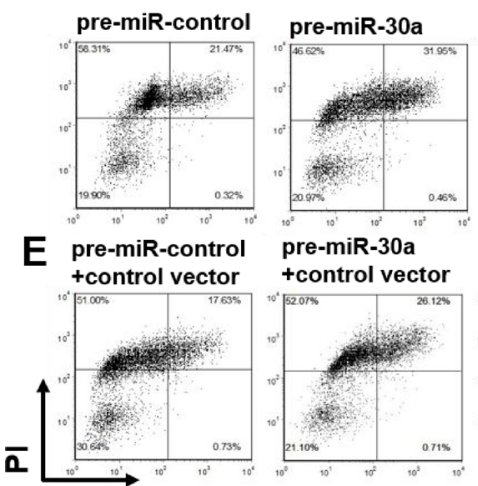

\section{Annexin V-FITC}
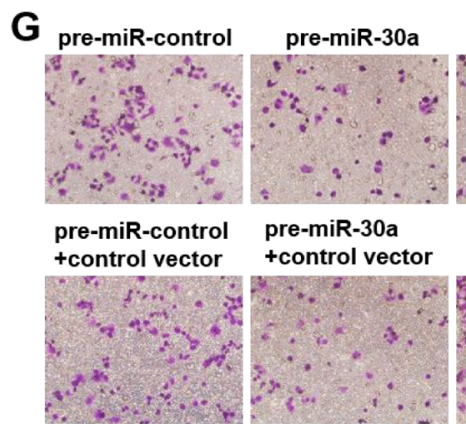
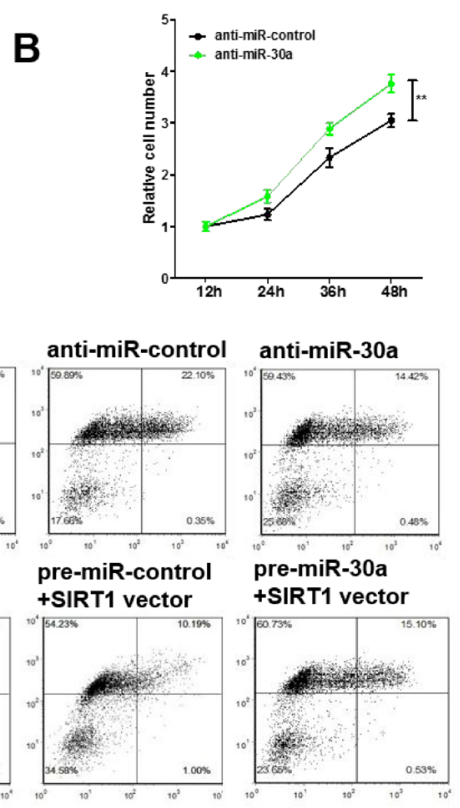

pre-miR-30a +SIRT1 vector

anti-miR-control anti-miR-30a
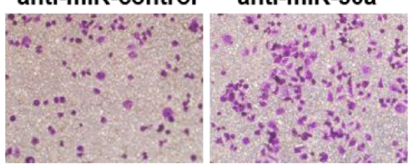

pre-miR-control +SIRT1 vector

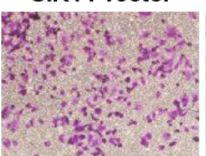

pre-miR-30a +SIRT1 vector

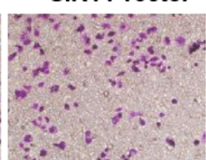

C
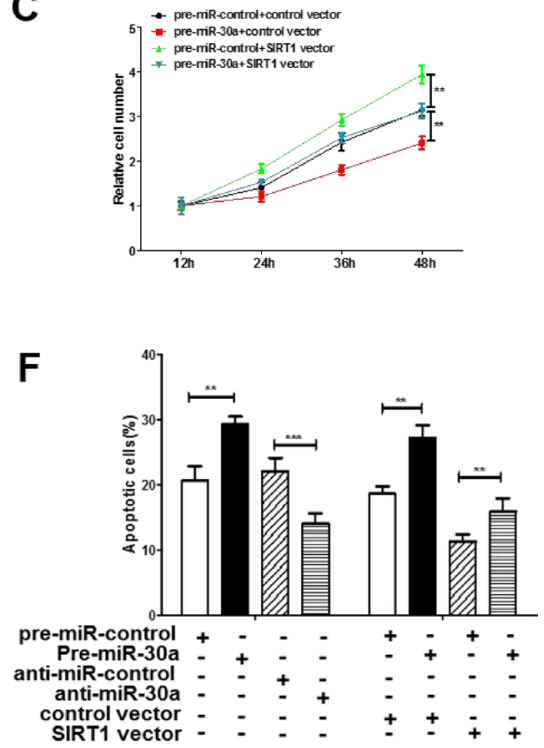

H

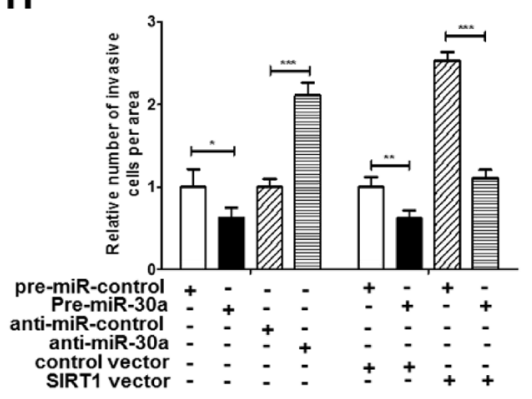

Figure 4: Effect of miR-30a and SIRT1 on the proliferation and apoptosis of lung cancer cells. (A) A cell proliferation assay was performed 12, 24, 36 and 48 hours after the transfection of A549 cells with equal doses of the miR-30a mimic or scrambled negative control RNA. (B) The cell proliferation assay was performed 12, 24, 36 and 48 hours after the transfection of A549 cells with equal doses of the miR-3a inhibitor or scrambled negative control RNA. (C) The cell proliferation assay was performed 12, 24, 36 and 48 hours after the transfection of A549 cells with equal doses of the pre-miR-control plus control plasmid, pre-miR-control plus SIRT1 overexpression plasmid, miR-30a mimic plus control plasmid, or miR-30a mimic plus SIRT1 overexpression plasmid. (D-F) The apoptosis assay was performed 24 hours after the transfection of A549 cells with equal doses of the miR-30a mimic, miR-30a inhibitor or scrambled negative control RNA or with equal doses of the pre-miR-control plus control plasmid, pre-miR-control plus SIRT1 overexpression plasmid, miR-30a mimic plus control plasmid, or miR-30a mimic plus SIRT1 overexpression plasmid. (D and E) representative image; (F) quantitative analysis. (G and $\mathbf{H})$ Transwell analysis was performed after the transfection of A549 cells with equal doses of the miR-30a mimic, miR-30a inhibitor or scrambled negative control RNA or with equal doses of the pre-miR-control plus control plasmid, pre-miR-control plus SIRT1 overexpression plasmid, miR-30a mimic plus control plasmid, or miR-30a mimic plus SIRT1 overexpression plasmid. G. representative image; H. quantitative analysis. The results are presented as the mean \pm S.E. of three independent experience $\left({ }^{*} p<0.05 ;{ }^{* *} p<0.01 ;{ }^{* * *} p<0.005\right)$. 
30a overexpression (Figure 5E), suggesting that SIRT1 overexpression is sufficient rescue the SIRT1 suppression caused by miR-30a. Furthermore, Hematoxylin and eosin (H\&E) staining of xenograft tissues showed more cell mitosis in the SIRT1-overexpressing group than the control group (Figure 5F). Xenografts from miR30a plus SIRT1 overexpression group exhibited less cell mitosis compared with xenografts with SIRT1 overexpression (Figure 5F and 5G), suggesting that miR30 a overexpression could attenuate the pro-proliferative effect of SIRT1. Immunohistochemical staining also revealed the lower levels of SIRT1 in tumors from mice implanted with miR-30a-overexpressing cells, whereas the tumors from the SIRT1-overexpressing mice showed increased SIRT1 protein levels (Figure 5F and 5G). Finally, the proliferative activity of tumor cells were assessed by immunocytochemistry with the mouse monoclonal antibody Ki-67. The cell proliferation rate measured by the percentage of Ki-67-positive tumor cells were increased in the group implanted with the SIRT1 plasmids and decreased in the group implanted with the miR-30a lentivirus (Figure 5F and 5G). Likewise, miR30a overexpression attenuated the pro-proliferative effect caused by SIRT1 overexpression (Figure 5F). These results validated the role of miR-30a in regulating lung cancer tumorigenesis.

\section{DISCUSSION}

Lung cancer is one of the most common cancers worldwide, and NSCLC constitutes approximately $80 \%$ of all primary lung cancers [33]. The efficacy of new treatments remains limited by a combination of drug resistance and our insufficient understanding of tumor cell signaling pathways. Many genes, including cancer inhibitors (tumor suppressors) and cancer inducers (oncogenes), influence lung carcinogenesis. SIRT1, a conserved nicotinamide adenine dinucteotide $(\mathrm{NAD}(+))$ dependent deacetylase, has been implicated in the occurrence and development of lung cancer. Noh et al.
A

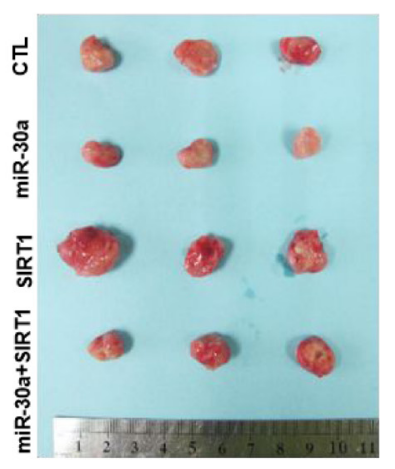

B

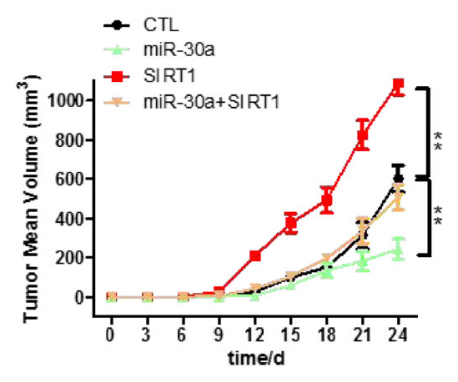

c

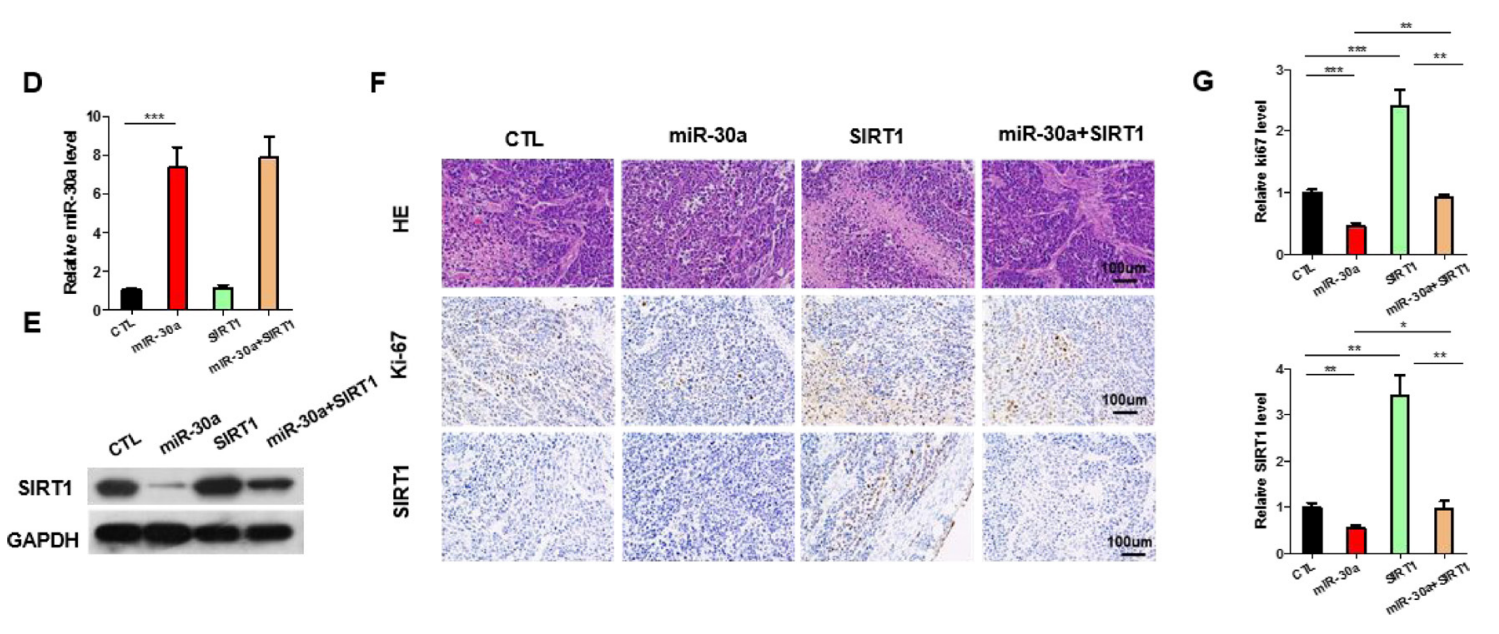

Figure 5: The function of miR-30a-SIRT1 axis on lung cancer growth in vivo. Mice were divided into four groups according to the implanted A549 cells: control cells (CTL), miR-30a overexpressing cells (miR-30a), SIRT1-overexpressing cells (SIRT1), and SIRT1 plus Lenti-miR-30a cells (miR-30a+ SIRT1). (A) Representative images of tumors. (B) The time course of tumor volume. (C) The quantitative analysis of tumor weight. (D) Quantitative RT-PCR analysis of the expression levels of miR-30a in tumors from four groups of mice. (E) Western blotting analyses of SIRT1 proteins in tumors from four groups of mice. (F) HE staining, Ki67 and SIRT1 immunohistochemical staining of tumor tissues in four groups of mice. (G) Quantitative analyses of Ki-67-positive and SIRT1-positive signals in the tumor. All data are shown as the means \pm S.E. obtained from three separate experiments. $\left({ }^{*} p<0.05 ;{ }^{* *} p<0.01 ;{ }^{* * *} p<0.005\right)$. 
confirmed that SIRT1 was significantly up-regulated in lung cancer [11]. Sun et al. showed that downregulation of SIRT1 by antisense oligonucleotides induces apoptosis and enhances radiation sensitization in lung cancer cells [34]. However, the precise role and regulatory mechanism of SIRT1 in the progression of lung cancer remain poorly understood.

In the present study, we observed that silencing SIRT1 expression through siRNA inhibits proliferation, invasion and promotes apoptosis in lung cancer cells, whereas overexpressing SIRT1 induced the opposite effects, suggesting a role for this protein as an essential oncogene during lung cancer progression. Interestingly, we identified an inconsistent trend between SIRT1 protein and mRNA levels in human lung cancer tissues. These results suggest a post-transcriptional regulation mechanism in regulating SIRT1 expression. One important mode of posttranscriptional regulation is the suppression of mRNA transcripts through miRNAs. Therefore, we searched for potential miRNAs that target SIRT1 and identified miR$30 \mathrm{a}$ as a candidate. Mechanistic studies revealed that miR30a could directly target the SIRT1 3'-UTR and inhibit its expression in lung cancer cells. Moreover, we showed that miR-30a inhibits SIRT1 expression and consequently suppresses proliferation and promotes apoptosis in lung cancer cells. These results indicate a novel regulatory axis in which miR-30a targeting SIRT1 regulates the proliferation and apoptosis of lung cancer cells.

Over the past decade, an important role for miRNAs in the genesis and progression of lung cancers has emerged $[35,36]$. In the present study, we observed that the level of miR-30a was lower in lung cancer tissues compared to matched adjacent noncancerous tissue. These results suggest that miR-30a might be involved in the tumorigenesis of lung cancer as a tumor suppressor. Indeed, miR-30a was downregulated in several types of cancers, including lung cancer [29, 37], breast cancer [38], prostate cancer [39] and gastric cancer [40] . Furthermore, miR-30a plays an anti-oncogenic role in cancer through the regulation of proliferation, migration, epithelialmesenchymal transition and cell adhesion [38, 41-43]. As was shown in Figure 6, we observed that overexpressing miR-30a inhibits proliferation and promote apoptosis in lung cancer cells, and silencing SIRT1 expression mimics miR-30a function in vitro and in vivo. Interestingly, we found that the restoration of SIRT1 expression successfully attenuates the anti-proliferative and pro-apoptotic effects of miR-30a on lung cancer cells, although miR-30a has many other targets. These results suggest that the targeting of SIRT1 is a main mechanism for miR-30 existing as a tumor suppressor in lung cancer.

Taken as a whole, this study describes a new regulatory axis that miR-30a possesses tumor suppressor activity by negatively regulating SIRT1 expression in lung tumorigenesis. This study may open new view for future lung cancer therapy.

\section{MATERIALS AND METHODS}

\section{Cells and human tissues}

The human lung cancer cell lines, A549 and H1975, were obtained from Shanghai Institute of Cell Biology, Chinese Academy of Sciences(Shanghai, China) and cultured in DMEM (Gibco, Carlsbad, CA, USA) supplemented with $10 \%$ fetal bovine serum within a humidified atmosphere containing $5 \% \mathrm{CO} 2$ at $37^{\circ} \mathrm{C}$. The lung tumors and paired normal adjacent tissues were derived from patients undergoing a surgical procedure at the Zhumadian Central Hospital (Zhumadian, China). All protocols concerning the use of patient samples in this study were approved by the Medical Ethics Committee of the Affiliated Zhumadian Central Hospital (Zhumadian, China). A signed consent form was obtained from each

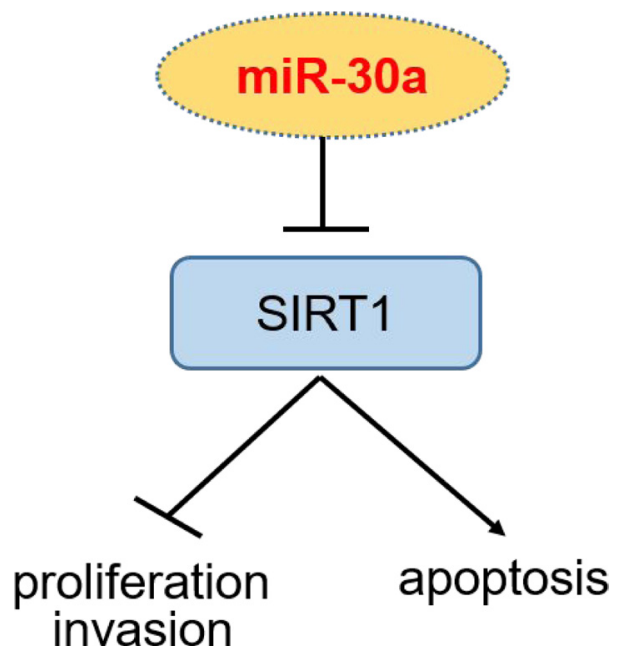

Figure 6: Schematic illustration of the miR-30a-SIRT1 axis. 
donor. The clinical features of the patients are listed in Supplementary Table 1.

\section{RNA isolation and quantitative RT-PCR}

Total RNA extraction, reverse transcription and TaqMan real-time polymerase chain reaction (PCR) for miRNAs were performed according to the manufacturer's instructions as described previously [44]. To quantify SIRT1 mRNA, $1 \mu \mathrm{g}$ of total RNA was reverse-transcribed to cDNA using oligo dT and Thermoscript (TaKaRa), performed using the following conditions: $42^{\circ} \mathrm{C}$ for $60 \mathrm{~min}$ and $85^{\circ} \mathrm{C}$ for $5 \mathrm{~min}$. SYBER Green Dye (Invitrogen), and specific primers for SIRT1 and GAPDH were used. The sequences of the primers were as follows: SIRT1 (sense): 5'-CTGTTTCCTGTGGGATACCTGACT-3'; SIRT1 (antisense): 5'-ATCGAACATGGCTTGAGGATCT-3'; GAPDH (sense): 5'-GATATTGTTGCCATCAATGAC-3'; GAPDH (antisense): 5'-TTGATTTTGGAGGGATCTC G-3'. The relative amount of SIRT1 mRNA was normalized to GAPDH.

\section{miRNA overexpression or knockdown}

Synthetic RNA molecules, including pre-miR-30a, anti-miR-30a and scrambled negative control RNAs (premiR-control and anti-miR-control) were purchased from GenePharma (Shanghai, China). The cells were seeded onto 6-well plates and transfected using Lipofectamine 2000 (Invitrogen) on the following day when the cells were approximately $70 \%$ confluent. In each well, equal 100 pmol of pre-miR-30a, anti-miR-30a or scrambled negative control RNA were used. The cells were harvested at $24 \mathrm{~h}$ after transfection for quantitative RT-PCR analysis and Western blotting.

\section{Plasmid construction and siRNA interference assay}

A mammalian expression plasmid encoding the human SIRT1 open reading frame (pReceiver-M02SIRT1) was purchased from GeneCopoeia (Germantown, MD, USA). An empty plasmid served as a negative control. The siRNAs (siRNA-1: 5'ACAGUUUCAUAGAGCCAUGAAGUAU-3', siRNA-2: ACUUUGCUGUAACCCUGUA) targeting human SIRT1 were purchased by GenePharma. A scrambled siRNA (GenePharma) was included as a negative control. Total RNA or protein was isolated $24 \mathrm{~h}$ or $48 \mathrm{~h}$ after transfection. The SIRT1 protein expression levels were assessed by Western blotting.

\section{Luciferase reporter assay}

A luciferase reporter assay was performed to check the direct binding of miR-30a to the target gene SIRT1 as previously described [45]. The normal and mutant
3'-UTR sequence of SIRT1 was directly synthesized by GenePharma and then inserted into PGL3 plasmid (Ambion). 293T cells were cultured in 24-well plates, and each well was transfected with $\beta$-galactosidase ( $\beta$-gal) expression plasmid (Ambion) and $0.2 \mu \mathrm{g}$ of firefly luciferase reporter plasmid, and equal amounts of pre-miR-30a and the scrambled negative control RNA using Lipofectamine 2000. The $\beta$-gal plasmid was used as a transfection control. After twenty-four hours, the cells were assayed using a luciferase assay kit (Promega, Madison, WI, USA).

\section{Western blotting}

The protein levels were analyzed by Western blot using the appropriate antibodies: anti-SIRT1 antibody (Abcam ab32441, Cambridge, MA, USA) and antiGAPDH (sc-365062; Santa Cruz Biotechnology, Santa Cruz, CA, USA), and were normalized by probing the same blots with an anti-GAPDH antibody. Protein bands were analyzed using the Image $\mathrm{J}$ software.

\section{Cell proliferation assay}

A549 cells were seeded in 96-well plates at a density of $1 \times 10^{4}$ cells per well and transfected 12 hours later. After transfection, $10 \mu \mathrm{l}$ WST- 8 solution from the CCK-8 kit (Beyotime, China) was added into each well. After incubation for 2 hours, the plates were read at 450 $\mathrm{nm}$ to measure the absorbance of each well (time 0 hour) and again at $12 \mathrm{~h}$ and $60 \mathrm{~h}$. The relative cell number was calculated as the ratio of absorbance of 12 hours to 60 hours.

\section{Cell invasion and apoptosis assays}

The invasion ability and apoptosis of A549 cells was tested in a Transwell Boyden Chamber $(6.5 \mathrm{~mm}$, Costar, Corning, NY, USA) and Annexin V-FITC/PI staining kit (BD Biosciences, San Diego, CA, USA ). Besides, the total apoptotic cells were counted as the sum of early apoptotic $(\mathrm{PI}-\mathrm{AV}+)$ and late apoptotic $(\mathrm{PI}+\mathrm{AV}+)$ cells.

\section{Establishment of tumor xenografts in mice}

Six-week-old male SCID (severe combined immune deficiency) mice (nu/nu) were purchased from the Model Animal Research Center of Nanjing University (Nanjing, China). Control A549 cells, miR-30a-overexpressing A549 cells, SIRT1- overexpressing A549 cells or miR-30a and SIRT1 co-overexpressing A549 cells were injected subcutaneously into SCID mice $\left(1 \times 10^{6}\right.$ cells per mouse, 3 mice per group). The mice were sacrificed after 25 days. The mouse lung tumors were removed, and the weight of the tumors was measured. Parts of the tissues were used for protein and total RNA extraction, and the remainder were fixed in $4 \%$ paraformaldehyde for $24 \mathrm{~h}$ and then 
processed for Hematoxylin and eosin (H\&E) staining and immunohistochemical staining for SIRT1 and Ki-67. All animal care and handling procedures were performed in accordance with the National Institutes of Health's Guide for the Care and Use of Laboratory Animals and were approved by the Institutional Review Board of Zhumadian Central Hospital (Zhumadian, China).

\section{Statistical analysis}

All Western blot images are representative of at least three independent experiments. Quantitative RTPCR, luciferase reporter assays, and cell proliferation and apoptosis assays were performed in triplicate, and each experiment was repeated several times. The data are shown as the means \pm SE of at least three independent experiments. The differences were considered statistically significant at $p<0.05$ using Student's $t$-test.

\section{Ethical approval}

This article does not contain any studies with human participants or animals performed by any of the authors.

\section{ACKNOWLEDGMENTS}

This work was supported by Educational Commission of Jiangxi Province of China (No. GJJ13036).

\section{CONFLICTS OF INTEREST}

The authors declare no conflict of interest.

\section{REFERENCES}

1. Jemal A, Bray F, Center MM, Ferlay J, Ward E, Forman D. Global Cancer Statistics. Ca-a Cancer Journal for Clinicians. 2011; 61:69-90. https://doi.org/10.3322/ caac. 20107.

2. Ramalingam SS, Owonikoko TK, Khuri FR. Lung cancer: New biological insights and recent therapeutic advances. CA Cancer J Clin. 2011; 61:91-112. https://doi.org/10.3322/ caac. 20102 .

3. Gatsonis CA, Natl Lung Screening Trial Res T. The National Lung Screening Trial: Overview and Study Design. Radiology. 2011; 258:243-53. https://doi. org/10.1148/radiol.10091808.

4. Bordone L, Guarente L. Calorie restriction, SIRT1 and metabolism: Understanding longevity. Nature Reviews Molecular Cell Biology. 2005; 6:298-305. https://doi. org/10.1038/nrm1616.

5. Liu T, Liu PY, Marshall GM. The Critical Role of the Class III Histone Deacetylase SIRT1 in Cancer. Cancer Research. 2009; 69:1702-5. https://doi.org/10.1158/0008-5472. can-08-3365.
6. Haigis MC, Sinclair DA. (2010). Mammalian Sirtuins: Biological Insights and Disease Relevance. Annual Review of Pathology-Mechanisms of Disease, pp. 253-95.

7. Tanner KG, Landry J, Sternglanz R, Denu JM. Silent information regulator 2 family of NAD-dependent histone/ protein deacetylases generates a unique product, 1-O-acetylADP-ribose. Proceedings of the National Academy of Sciences of the United States of America. 2000; 97:1417882. https://doi.org/10.1073/pnas.250422697.

8. Zhang Y, Zhang M, Dong H, Yong S, Li X, Olashaw N, Kruk PA, Cheng JQ, Bai W, Chen J, Nicosia SV, Zhang $\mathrm{X}$. Deacetylation of cortactin by SIRT1 promotes cell migration. Oncogene. 2009; 28:445-60. https://doi. org/10.1038/onc.2008.388.

9. Huffman DM, Grizzle WE, Bamman MM, Kim JS, Eltoum IA, Elgavish A, Nagy TR. SIRT1 is significantly elevated in mouse and human prostate cancer. Cancer Research. 2007; 67:6612-8. https://doi.org/10.1158/0008-5472.can-07-0085.

10. Jang KY, Kim KS, Hwang SH, Kwon KS, Kim KR, Park HS, Park BH, Chung MJ, Kang MJ, Lee DG, Moon WS. Expression and prognostic significance of SIRT1 in ovarian epithelial tumours. Pathology. 2009; 41:366-71. https://doi. org/10.1080/00313020902884451.

11. Noh SJ, Baek HA, Park HS, Jang KY, Moon WS, Kang MJ, Lee DG, Kim MH, Lee JH, Chung MJ. Expression of SIRT1 and cortactin is associated with progression of non-small cell lung cancer. Pathology Research and Practice. 2013; 209:365-70. https://doi.org/10.1016/j.prp.2013.03.011.

12. Luo JY, Nikolaev AY, Imai S, Chen DL, Su F, Shiloh A, Guarente L, Gu W. Negative control of $\mathrm{p} 53$ by Sir2 alpha promotes cell survival under stress. Cell. 2001; 107:137-48. https://doi.org/10.1016/s0092-8674(01)00524-4.

13. Brooks $\mathrm{CL}, \mathrm{Gu}$ W. How does SIRT1 affect metabolism, senescence and cancer? Nature Reviews Cancer. 2009; 9:123-8. https://doi.org/10.1038/nrc2562.

14. Firestein R, Blander G, Michan S, Oberdoerffer P, Ogino S, Campbell J, Bhimavarapu A, Luikenhuis S, de Cabo R, Fuchs C, Hahn WC, Guarente LP, Sinclair DA. The SIRT1 Deacetylase Suppresses Intestinal Tumorigenesis and Colon Cancer Growth. Plos One. 2008; 3. https://doi.org/10.1371/ journal.pone.0002020.

15. Lin CJ, Gong HY, Tseng HC, Wang WL, Wu JL. miR122 targets an anti-apoptotic gene, Bcl-w, in human hepatocellular carcinoma cell lines. Biochem Biophys Res Commun. 2008; 375:315-20. https://doi.org/10.1016/j. bbrc.2008.07.154.

16. Nemoto S, Fergusson MM, Finkel T. Nutrient availability regulates SIRT1 through a forkhead-dependent pathway. Science. 2004; 306:2105-8. https://doi.org/10.1126/ science. 1101731 .

17. Chen WY, Wang DH, Yen RWC, Luo JY, Gu W, Baylin SB. Tumor suppressor HIC1 directly regulates SIRT1 to modulate p53-dependent DNA-damage responses. Cell. 2005; 123:437-48. 
18. Bartel DP. MicroRNAs: Genomics, biogenesis, mechanism, and function. Cell. 2004; 116:281-97. https://doi. org/10.1016/s0092-8674(04)00045-5.

19. Kim VN. MicroRNA biogenesis: Coordinated cropping and dicing. Nature Reviews Molecular Cell Biology. 2005; 6:376-85. https://doi.org/10.1038/nrm1644.

20. Wu L, Belasco JG. Let me count the ways: Mechanisms of gene regulation by miRNAs and siRNAs. Molecular Cell. 2008; 29:1-7. https://doi.org/10.1016/j.molcel.2007.12.010.

21. Calin GA, Croce CM. MicroRNA signatures in human cancers. Nature Reviews Cancer. 2006; 6:857-66. https:// doi.org/10.1038/nrc1997.

22. Ma L, Weinberg RA. Micromanagers of malignancy: role of microRNAs in regulating metastasis. Trends in Genetics. 2008; 24:448-56. https://doi.org/10.1016/j.tig.2008.06.004.

23. Jin F, Wang Y, Zhu Y, Li S, Liu Y, Chen C, Wang X, Zen K, Li L. The miR-125a/HK2 axis regulates cancer cell energy metabolism reprogramming in hepatocellular carcinoma. Scientific Reports. 2017; 7. https://doi.org/10.1038/ s41598-017-03407-3.

24. Guan P, Yin ZH, Li XL, Wu W, Zhou BS. Meta-analysis of human lung cancer microRNA expression profiling studies comparing cancer tissues with normal tissues. Journal of Experimental \& Clinical Cancer Research. 2012; 31. https:// doi.org/10.1186/1756-9966-31-54.

25. Lewis BP, Shih IH, Jones-Rhoades MW, Bartel DP, Burge CB. Prediction of mammalian microRNA targets. Cell. 2003; 115:787-98.

26. John B, Enright AJ, Aravin A, Tuschl T, Sander C, Marks DS. Human MicroRNA targets. PLoS Biol. 2004; 2: e363. https://doi.org/10.1371/journal.pbio.0020363.

27. Krek A, Grun D, Poy MN, Wolf R, Rosenberg L, Epstein EJ, MacMenamin P, da Piedade I, Gunsalus KC, Stoffel M, Rajewsky N. Combinatorial microRNA target predictions. Nat Genet. 2005; 37:495-500. https://doi.org/10.1038/ ng1536.

28. He RQ, Yang LH, Lin XM, Chen X, Lin XG, Wei FL, Liang XN, Luo YH, Wu YZ, Gan TQ, Dang YW, Chen G. MiR-30a-5p suppresses cell growth and enhances apoptosis of hepatocellular carcinoma cells via targeting AEG-1. International Journal of Clinical and Experimental Pathology. 2015; 8:15632-41.

29. Wen XP, Ma HL, Zhao LY, Zhang W, Dang CX. MiR-30a suppresses non-small cell lung cancer progression through AKT signaling pathway by targeting IGF1R. Cellular and Molecular Biology. 2015; 61:78-85.

30. Li LS, Yuan LJ, Luo JM, Gao J, Guo JL, Xie XM. MiR34a inhibits proliferation and migration of breast cancer through down-regulation of Bcl-2 and SIRT1. Clinical and Experimental Medicine. 2013; 13:109-17. https://doi. org/10.1007/s10238-012-0186-5.
31. Yang A, Wang X, Yul C, Jin Z, Wei L, Ca J, Wang Q, Zhang M, Zhang L, Zhang L, Hao C. microRNA-494 is a potential prognostic marker and inhibits cellular proliferation, migration and invasion by targeting SIRT1 in epithelial ovarian cancer. Oncology Letters. 2017; 14:3177-84. https://doi.org/10.3892/ol.2017.6501.

32. Yamakuchi M, Ferlito M, Lowenstein CJ. miR-34a repression of SIRT1 regulates apoptosis. Proceedings of the National Academy of Sciences of the United States of America. 2008; 105:13421-6. https://doi.org/10.1073/ pnas.0801613105.

33. Parkin DM. Global cancer statistics in the year 2000. Lancet Oncology. 2001; 2:533-43. https://doi.org/10.1016/ s1470-2045(01)00486-7.

34. Sun YN, Sun DC, Li F, Tian LL, Li CR, Li L, Lin RX, Wang SQ. Downregulation of Sirt1 by antisense oligonucleotides induces apoptosis and enhances radiation sensitization in A549 lung cancer cells. Lung Cancer. 2007; 58:21-9. https://doi.org/10.1016/j.lungcan.2007.05.013.

35. Garzon R, Calin GA, Croce CM. (2009). MicroRNAs in Cancer. Annual Review of Medicine, pp. 167-79.

36. Yanaihara N, Caplen N, Bowman E, Seike M, Kumamoto K, Yi M, Stephens RM, Okamoto A, Yokota J, Tanaka T, Colin GA, Liu CG, Croce CM, et al. Unique microRNA molecular profiles in lung cancer diagnosis and prognosis. Cancer Cell. 2006; 9:189-98. https://doi.org/10.1016/j. ccr.2006.01.025.

37. Zhu JJ, Zeng YY, Xu C, Qin HL, Lei Z, Shen D, Liu ZY, Huang JA. Expression profile analysis of microRNAs and downregulated miR-486-5p and miR-30a-5p in non-small cell lung cancer. Oncology Reports. 2015; 34:1779-86. https://doi.org/10.3892/or.2015.4141.

38. Fu J, Xu XJ, Kang L, Zhou LY, Wang SB, Lu JM, Cheng L, Fan ZY, Yuan B, Tian PR, Zheng XF, Yu CZ, Ye QN, et al. miR-30a suppresses breast cancer cell proliferation and migration by targeting Eya2. Biochemical and Biophysical Research Communications. 2014; 445:314-9. https://doi. org/10.1016/j.bbrc.2014.01.174.

39. Xu CG, Yang MF, Fan JX, Wang W. MiR-30a and miR-205 are downregulated in hypoxia and modulate radiosensitivity of prostate cancer cells by inhibiting autophagy via TP53INP1. European Review for Medical and Pharmacological Sciences. 2016; 20:1501-8.

40. Wang LL, Zhang XH, Zhang X, Chu JK. MiR-30a increases cisplatin sensitivity of gastric cancer cells through suppressing epithelial-to-mesenchymal transition (EMT). European Review for Medical and Pharmacological Sciences. 2016; 20:1733-9.

41. Zhu H, Wu H, Liu XP, Li BA, Chen Y, Ren XC, Liu CG, Yang JM. Regulation of autophagy by a beclin 1-targeted microRNA, miR-30a, in cancer cells. Autophagy. 2009; 5:816-23. 
42. Liu ZF, Chen L, Zhang XC, Xu X, Xing HX, Zhang YJ, Li WJ, Yu H, Zeng JP, Jia JH. RUNX3 regulates vimentin expression via miR-30a during epithelial- mesenchymal transition in gastric cancer cells. Journal of Cellular and Molecular Medicine. 2014; 18:610-23. https://doi. org/10.1111/jcmm. 12209.

43. Li WT, Liu CL, Zhao CL, Zhai LM, Lv SJ. Downregulation of beta 3 integrin by miR-30a-5p modulates cell adhesion and invasion by interrupting Erk/Ets-1network in triplenegative breast cancer. International Journal of Oncology. 2016; 48:1155-64. https://doi.org/10.3892/ijo.2016.3319.
44. Jin F, Wang Y, Li M, Zhu Y, Liang H, Wang C, Wang F, Zhang CY, Zen K, Li L. MiR-26 enhances chemosensitivity and promotes apoptosis of hepatocellular carcinoma cells through inhibiting autophagy. Cell Death \& Disease. 2017; 8. https://doi.org/10.1038/cddis.2016.461.

45. Chen X, Guo X, Zhang H, Xiang Y, Chen J, Yin Y, Cai X, Wang K, Wang G, Ba Y, Zhu L, Wang J, Yang R, et al. Role of miR-143 targeting KRAS in colorectal tumorigenesis. Oncogene. 2009; 28:1385-92. https://doi.org/10.1038/ onc.2008.474. 